\title{
Detection of Toxoplasma gondii-specific Antibodies in Dogs. A Comparative Study of Immunoenzymatic, Immunofluorescent and Haemagglutination Titers
}

\author{
Deise AO Silva, Dagmar D Cabral ${ }^{*}$, Bernardette LD Bernardina*, \\ Maria A Souza, José R Mineo/ ${ }^{+}$
}

\begin{abstract}
Laboratório de Imunologia *Laboratório de Parasitologia, Departamento de Patologia, Universidade Federal de Uberlândia, Av. Pará 1720, Campus Umuarama, 38400-902 Uberlândia, MG, Brasil
\end{abstract}

We evaluated the titers of anti-T. gondii antibodies by various serological tests in 40 serum samples from dogs exhibiting clinical signs of infectious diseases. Indirect immunofluorescence (IgG-IFI), indirect haemagglutination (IHA and M-Toxo) and immunoenzymatic (ELISA and PA-ELISA) tests were carried out. Titers $\geq 64$ were considered as positive. Anti-Toxoplasma antibodies were found in $9(22.5 \%)$, $14(35 \%), 14(35 \%)$ and $12(30 \%)$ samples, respectively for IHA, IgG-IFI, ELISA and PA-ELISA. The results showed that $57 \%$ were negative in all tests and $43 \%$ of the dogs presented antibodies to $\mathrm{T}$. gondii; from these, $20 \%$ were positive in all three tests with high titers of antibodies and $23 \%$ were positive in only one or two tests with low titers of antibodies and mainly related to the IFI and ELISA tests. We observed $5(12.5 \%)$ and $1(2.5 \%)$ reactive samples, respectively, by M-Toxo and IHA with or without 2mercapthoethanol, in the attempt to detect specific IgM. We can conclude that serodiagnosis of toxoplasmosis in dog have to be based on the combination of serological tests (IFI and ELISA) and with emphasis at the determination of the titers and the classes of the specific antibodies.

Key words: Toxoplasma gondii - dogs - antibodies - indirect immunofluorescence - indirect haemagglutination ELISA

Toxoplasma gondii causes one of the most common parasitic infections in the world, affecting a wide range of hosts including man, domestic animals and birds. Toxoplasma infection in dogs is very common as demonstrated by various serological surveys (Svoboda 1987, Lindsay et al. 1990, Uggla et al. 1990, Ulón \& Marder 1990, Guimarães et al. 1992, Shad-Del et al. 1993, Björkman et al. 1994) while clinical toxoplasmosis is very less frequent, usually seen in young animals, and is associated with concurrent immunosupressor factors or infections, as distemper virus (Dubey 1985).

The clinical signs of toxoplasmosis in dogs are usually characterized by ataxia, diarrhoea and respiratory distress (Ahmed et al. 1983). Focal necrotic areas in the lung, liver and brain of infected dogs are common and could lead to development of several clinical signs. Therefore, toxoplasmosis in dogs and other animals can mimic many infectious diseases (Dubey 1985).

Serological diagnosis of $T$. gondii infections in dogs has been evaluated by many investigators

${ }^{+}$Corresponding author. Fax: +55-34-218.2333

Received 3 January 1997

Accepted 23 June 1997
(Ahmed et al. 1983, Svoboda 1987, Lindsay et al. 1990, Uggla et al. 1990, Ulón \& Marder 1990, Hejlicek et al. 1995). The tests used include the Sabin-Feldman, the complement fixation, the indirect haemagglutination, the direct agglutination, the indirect fluorescent antibody and the enzyme immunoassay. The demonstration of antibodies by these serological tests just indicates previous infection by $T$. gondii. A laboratory diagnosis defined to toxoplasmosis-disease requires the demonstration of high titers of specific antibodies and increasing levels in two serum samples taken 2 to 4 weeks apart (Dubey 1987). The majority of these investigations carried out in dogs have not established a comparative study of the titers of these various tests and their possible correlation with active infection by $T$. gondii.

The purpose of the present investigation was to evaluate the titers of the various serological tests conducted with serum samples from dogs exhibiting clinical signs of infectious processes in which toxoplasmosis was one of the presumable diseases.

\section{MATERIALS AND METHODS}

Animals and serum samples - A total of 40 serum samples were obtained from dogs presented to the Veterinary Hospital, Universidade Federal de Uberlândia, State of Minas Gerais, Brazil, from 
1990 to 1993, with clinical signs which might have led clinicians to suspect infection with $T$. gondii: apathy, fever, dyspnea, pneumonia, gastroenteritis and nervous system disturbances with incoordination, tremors and paralysis. The dogs were of various pure and mixed breed ancestry. Their ages varied from a few weeks to 14 years (mean of $2.9 \pm$ 0.6 years) and the numbers of male and female were 19 and 21, respectively. The serum samples were collected following centrifugation at $500 \mathrm{x} \mathrm{g}$ of 5 $\mathrm{ml}$ of blood obtained from the radial vein from the dogs and were stored at $-20^{\circ} \mathrm{C}$ until analyzed for Toxoplasma antibodies.

Serological tests - An indirect haemagglutination test (IHA) was performed using a reagent commercially available (Hematoxo, Biolab Diagnostica S.A., São Paulo, Brazil). The serum samples were diluted twofold, from 1:64 to 1:2,048, following the steps described by the manufacturer. All positive serum samples in the IHA test were retested after treatment with 2-Mercaptoethanol (2$\mathrm{ME})$ in order to verify the presence of IgM antibodies (Camargo et al. 1978). Other IHA (M-Toxo) developed by Yamamoto et al. (1991) for the serodiagnosis of acute toxoplasmosis was carried out with a standardized suspension of red blood cells coated with a heat-stable alkaline-solubilized extract of $T$. gondii, which reacts predominantly with IgM antibodies. To $50 \mu \mathrm{l}$ of doubling diluted serum, as above mentioned, $25 \mu 1$ of sensitized cell suspensions were added and the agglutination pattern read after incubation of $1 \mathrm{hr}$ and $30 \mathrm{~min}$ at room temperature. Positive and negative reference serum samples (for IgG and IgM specific to $T$. gondii) were included in all assays.

The indirect immunofluorescence test for detection of IgG antibodies to $T$. gondii ( $I g G-I F I)$ used in this investigation was similar to that used for diagnosis of human infections (Camargo 1964). Antigen slides of $T$. gondii were incubated with serum samples screened at 1:64 dilution and positive samples were then diluted twofold until 1: 4,096. An isothiocyanate fluorescein labeled rabbit IgG anti-dog IgG (kindly supplied by Centro de Zoonoses, São Paulo, Brazil) was used as secondary antibody and the optimum titer $(1: 150)$ was determined by block titration with positive and negative serum controls. Positive control sera were obtained of the dogs with consistently positive serological results by IHA-Hematoxo, in interassay and intraassay variation tests. Negative control sera were obtained of dogs healthy with consistently negative serological results by IHA-Hematoxo, as mentioned above. The slides were examined by epifluorescent microscope (Olympus, Mod. BH2, Tokyo, Japan).
An immunoenzymatic test (ELISA) was carried out for detection of $\operatorname{IgG}$ antibodies anti- $T$. gondii as described (Mineo et al. 1980) with some modifications. Microtiter plates (Hemobag, Campinas, Brazil) were coated overnight at $4{ }^{\circ} \mathrm{C}$ with a soluble antigen preparation $(0.25 \mu \mathrm{g}$ of protein/well), consisting of a sonicated extract of purified Toxoplasma tachyzoites diluted in $0.06 \mathrm{M}$ sodium carbonate buffer ( $\mathrm{pH}$ 9.6). The plates were washed three times with phosphate-buffered saline containing $0.05 \%$ Tween 20 (PBST) and incubated with the samples. Doubling dilutions of the dog serum samples, diluted from 1:64 to 1:16,384 in PBST with $20 \%$ equine serum (PBST/ES) were added in duplicates to the wells and the plates incubated at $37^{\circ} \mathrm{C}$ for $45 \mathrm{~min}$. After repeated washing, the secondary antibody, consisting of a peroxidase (horseradish peroxidase, type VI, Sigma Co., St. Louis, USA) labeled to rabbit IgG antidog IgG, prepared as described (Wilson \& Nakane 1978) and diluted 1:20,000 in PBST/ES, was added and incubation performed for $45 \mathrm{~min}$ at $37^{\circ} \mathrm{C}$. After a final wash, the plates were incubated with enzyme substrate hydrogen peroxide and o-phenylenediamine (Merck, Germany) in $0.1 \mathrm{M}$ citrate$\mathrm{Na}_{2} \mathrm{HPO}_{4}$ buffer (pH 5.5) for $15 \mathrm{~min}$ at room temperature. The reaction was stopped by adding $2 \mathrm{~N}$ $\mathrm{H}_{2} \mathrm{SO}_{4}$ and the absorbance was read at $492 \mathrm{~nm}$ using a Titertek Multiskan-Plus spectrophotometer (Flow Laboratories, USA). Positive and negative serum controls previously tested by conventional serological tests (i.e., IHA and IFI) were included on each plate. Samples showing absorbance values exceeding the mean absorbance of the negative controls plus 2 standard deviations were considered as positive. Other immunoenzymatic test (PA-ELISA) was developed for detection of $\operatorname{IgG}$ antibodies anti- $T$. gondii, as described above, except by using peroxidase labeled Protein A (Sigma Co., St. Louis, USA) as secondary antibody which was diluted 1:100,000 in PBST with 1\% bovine serum albumin.

The titers obtained on the serological tests were defined as low titers when the reactivity was equal or lower than 128. On the other hand, titers higher than 128 were defined as high titers.

Statistical analyses - The antibody titers were codified in $\log _{10}$ in order to determine the geometric means of the titers (GMT) (White 1973). The significance of the difference among these values was determined by the Mann-Whitney and Kruskal-Wallis' tests (White 1973). The correlation coefficients between the titers provided by the IHA, IgG-IFI, ELISA and PA-ELISA tests were determined as reported (Lutz 1967). 


\section{RESULTS}

When carrying out the indirect immunofluorescence (IgG-IFI), indirect haemagglutination (IHA and M-Toxo) and immunoenzymatic (ELISA and PA-ELISA) tests, the criteria followed for the samples be considered positive was the detection of antibodies in titers $\geq 64$. The IHA test revealed T. gondii antibodies in 9 samples $(22.5 \%)$; the IgGIFI test demonstrated specific antibodies in 14 samples (35\%), whereas the ELISA and PA-ELISA tests showed anti-Toxoplasma antibodies in 14 $(35 \%)$ and $12(30 \%)$, respectively. In the attempt to detect specific IgM by the IHA with $2 \mathrm{ME}$ and M-Toxo tests, it was observed $1(2.5 \%)$ and 5 $(12.5 \%)$ reactive samples, respectively. The distribution of Toxoplasma antibody titers determined by all these serological tests is demonstrated in Table. We observed titers of 64 to 2,048 for IgGIFI $($ GMT $=2.68)$; titers of 64 to 8,192 for both ELISA and PA-ELISA (GMT $=3.43$ and 3.22, respectively) and titers of 64 to 512 for IHA (GMT $=2.54$ ).

Based on these results, it was possible to divide the positive samples in two different groups: a) a group of positive samples in all three tests (from \# 1 to \# 8) which presented high titers of antibodies; and b) a group of positive samples in only one or two tests (from \# 9 to \# 17) which revealed low titers of antibodies. The positive results of this latter group were mainly related to the IFI and ELISA tests. The GMTs for the high titer group in comparison with the low one were 2.87 and $2.07 ; 3.67$ and $1.98 ; 3.39$ and $1.90 ; 2.57$ and 2.11, respectively for IgG-IFI, ELISA, PA-ELISA and IHA $(\mathrm{p}<0.01)$. In addition, the reactive samples for the IHA with $2 \mathrm{ME}$ and M-Toxo tests (GMT $=2.56$ and 2.32, respectively) were predominantly included in the group of positive samples with high titers. No significant differences were seen according to sex, age and ancestry.

The correlation coefficient between the titers provided by ELISA versus PA-ELISA was highly significant (.98; $\mathrm{p}<0.01)$. On the other hand, the comparison between IHA and IgG-IFI shows a non-significant correlation index of .51 ( $p>0.05)$, while the comparison between ELISA versus IgGIFI and IHA versus ELISA demonstrated significant correlation indexes (.69 and .81, respectively; $\mathrm{p}<0.01)$.

Fig. summarizes the results found in this investigation. It was observed $20 \%(8 / 17)$ of positivity in all three tests, $23 \%(9 / 17)$ in only one or

TABLE

Distribution of Toxoplasma antibody titers determined by indirect immunofluorescence test (IgG-IFI), immunoenzymatic test (ELISA and PA-ELISA) and indirect haemagglutination test (IHA, IHA-w2ME, M-Toxo) among 17 positive samples from 40 serum samples collected from dogs with clinical signs of infectious diseases as examined at Veterinary Hospital of Universidade Federal de Uberlândia, State of Minas Gerais, Brazil (1990-1993)

\begin{tabular}{|c|c|c|c|c|c|c|}
\hline \multirow{3}{*}{$\begin{array}{l}\text { Samples } \\
\text { no. }\end{array}$} & \multicolumn{6}{|c|}{ Antibody titers } \\
\hline & \multirow{2}{*}{$\begin{array}{c}\text { IFI } \\
\text { IgG-IFI }\end{array}$} & \multicolumn{2}{|c|}{ ELISA } & \multicolumn{3}{|c|}{ IHA } \\
\hline & & ELISA & PA-ELISA & IHA & IHA-w2ME & M -Toxo \\
\hline 1 & 128 & 8192 & 4096 & 512 & 512 & 128 \\
\hline 2 & 128 & 4096 & 1024 & 256 & 128 & - \\
\hline 3 & 2048 & 8192 & 8192 & 512 & 512 & 512 \\
\hline 4 & 256 & 2048 & 1024 & 512 & 256 & 64 \\
\hline 5 & 1024 & 4096 & 2048 & 64 & - & - \\
\hline 6 & 256 & 2048 & 1024 & 512 & 256 & - \\
\hline 7 & 2048 & 8192 & 2048 & 512 & 512 & 128 \\
\hline 8 & 64 & 512 & 256 & 128 & - & - \\
\hline 9 & - & 64 & - & - & - & - \\
\hline 10 & 128 & - & - & 128 & 64 & 64 \\
\hline 11 & 256 & 128 & 128 & - & - & - \\
\hline 12 & 64 & 64 & 64 & - & - & - \\
\hline 13 & 64 & - & - & - & - & - \\
\hline 14 & - & 128 & 64 & - & - & - \\
\hline 15 & 64 & - & - & - & - & - \\
\hline 16 & 128 & 128 & 64 & - & - & - \\
\hline 17 & - & 64 & - & - & - & - \\
\hline
\end{tabular}

Cut-off $\geq 64$ 
two tests, establishing a total of $43 \%$ (17/40) of seropositive samples for $T$. gondii antibodies, and $57 \%$ (23/40) of negativity in all tests.

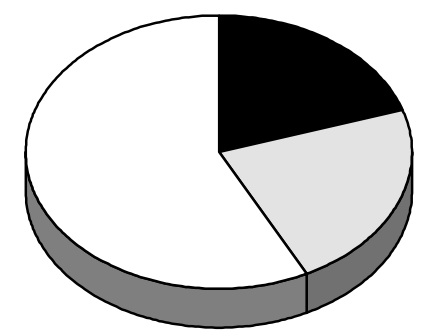

Percentage of negativity or positivity for Toxoplasma gondii antibodies as determined by indirect haemagglutination test (IHA), indirect immunofluorescence test (IgG-IFI) and immunoenzymatic test (ELISA and PA-ELISA) among $40 \mathrm{se}-$ rum samples collected from dogs with clinical signs of infectious diseases as examined at Veterinary Hospital of Universidade Federal de Uberlândia, State of Minas Gerais, Brazil (1990-1993).

\section{DISCUSSION}

Many investigations have been conducted on serological diagnosis of $T$. gondii infection in dogs. However, the majority of them have not established a comparative study of the antibody titers and their possible correlation with active infection by $T$. gondii. So far, it has not been possible to make valid comparisons among prevalence of antibody to $T$. gondii in serum samples of dogs from these various studies because of the differences in sensitivity and specificity of the employed serological tests and also the lack of standardization for each test (Dubey 1985).

The variations in incidence of Toxoplasma serum titers could be related to modifications in infection rates or to the sensitivity of the different tests used as well as the cut-off established for each test (Ahmed et al. 1983). In this context, it should also be pointed out that the prevalence of animals with $T$. gondii antibodies is closely dependent on the titer regarded as positive. In most studies the usual dilutions of 1:16, 1:64 or 1:100 (for IFI, IHA or ELISA, respectively) were considered as positive (Ahmed et al. 1983, Svoboda 1987, Ugla et al. 1990).

The present study is the first publication concerning the comparative analyses of the IFI, IHA and ELISA tests in dogs. Therefore, we evaluate the titers of anti-T. gondii antibodies determined by three serological tests conducted with serum samples from dogs exhibiting clinical signs suggestive of toxoplasmosis.

Our results showed that, even though all serum samples came from dogs exhibiting clinical signs of infectious disease compatible with toxoplasmosis, only $43 \%$ of them presented antibodies against $T$. gondii. Thus, three groups were identified: (1) seronegative samples (57\%) - this finding can be explained by the fact that there is an overlapping of clinical signs among toxoplasmosis and many infectious diseases in dogs and other animals, so that they can mimic each other (Ahmed et al. 1983). Thus, a negative serological result generally rules out a diagnosis of toxoplasmosis and shows significance, as it may guide the procedures of the clinicians in research for other diseases; (2) seropositive samples in all three tests (20\%) with high titers of antibodies and predominance of IgM antibodies detected mainly by the M-Toxo test. This test, developed for the serodiagnosis of acute human toxoplasmosis (Yamamoto et al. 1991), was carried out with the dog serum samples in order to verify its suitability for detection of IgM-specific antibodies in dogs. It was found 4 positive samples by M-Toxo out of 8 positive samples (50\%) for IgG antibodies in all three tests, suggesting that these animals might be presenting an active infection by $T$. gondii. The $4 \mathrm{M}$-Toxo non-reactive samples in this group but with high titers of $\mathrm{IgG}$ may represent IgM false-negative results due to competitive interaction with $\operatorname{IgG}$ which presents higher avidity; and (3) seropositive samples in only 1 or 2 tests $(23 \%)$ with low titers of antibodies and mainly related to the IFI and ELISA tests. The results of this group suggest that the samples might belong to animals with chronic infection and the titer and the positivity rates were totally dependent of the sensibility of the employed tests. From the serological methods used in the present study, ELISA and IFI can be strongly recommended. On the other hand, by using IHA, a negative result should be taken carefully because the possibility of $T$. gondii infection cannot be excluded.

It was shown in the present investigation high correlation among the ELISA and PA-ELISA tests. Since it seems that the use of Protein A - peroxidase in the ELISA test for the determination of Toxoplasma antibodies in dogs has not previously been reported, we concluded that PA-ELISA could also be used in serological surveys in dogs for detection of anti-T. gondii $\operatorname{IgG}$ antibodies.

Taken together, the results presented here show that the diagnosis of toxoplasmosis in dog have to be based on combination of serological tests that are able to detect different anti- $T$. gondii antibodies and with emphasis in the determination of the titers and the classes of the immunoglobulins. 


\section{REFERENCES}

Ahmed BA, Gaafar SM, Weirich WE, Kanitz CL 1983. Relationship of Toxoplasma infections to other diseases in dogs. Vet Parasitol 12: 199-203.

Björkman C, Lundén A, Uggla A 1994. Prevalence of antibodies to Neospora caninum and Toxoplasma gondii in Swedish dogs. Acta Vet Scand 35: 445447.

Camargo ME 1964. Improved technique of indirect immunofluorescence for serological diagnosis of toxoplasmosis. Rev Inst Med Trop S Paulo 6: 117-118.

Camargo ME, Ferreira AW, Mineo JR, Takiguti CK, Nakahara OS 1978. Immunoglobulin G and immunoglobulin M enzyme-linked immunosorbent assays and defined toxoplasmosis serological titers. Infect Immun 21: 35-38.

Dubey JP 1985. Toxoplasmosis in dogs. Canine Pract 12: 7-28.

Dubey JP 1987. Toxoplasmosis. Vet Clin North Am Small Animal Practice 17: 1389-1404.

Guimarães AM, Ribeiro MFB, Lima JD, Cury MC, Spiewak G 1992. Freqüência de anticorpos antiToxoplasma gondii em cães de Belo Horizonte, Minas Gerais. Arq Bras Med Vet Zootec 44: 67-68.

Hejlicek K, Literak I, Lhotak M 1995. Prevalence of antibodies to Toxoplasma gondii in army dogs in the Czech Republic and Slovak Republic. Vet Medicina 40: 137-140.

Lindsay DS, Dubey JP, Upton SJ, Ridley RK 1990. Serological prevalence of Neospora caninum and Toxoplasma gondii in dogs from Kansas. J Helminthol Soc Wash 57: 86-88.
Lutz W 1967. Statistical methods applied to immunological data, p. 1163-1202. In DM Weir, Handbook of Experimental Immunology, Blackwell, Oxford.

Mineo JR, Camargo ME, Ferreira AW 1980. Enzymelinked immunosorbent assay for antibodies to Toxoplasma gondii polysaccharides in human toxoplasmosis. Infect Immun 27: 283-287.

Shad-Del F, Sarvestani RG, Milani MS 1993. Sero-prevalence of Toxoplasma infection in human and dog population in Shiraz, Iran. J Appl An Research 3: 83-89.

Svoboda M 1987. Incidence of Toxoplasma gondii antibodies in dogs from Brno and its environs. Acta Vet Brno 56: 475-486.

Uggla A, Mattson S, Juntti N 1990. Prevalence of antibodies to Toxoplasma gondii in cats, dogs and horses in Sweden. Acta Vet Scand 31: 219-222.

Ulón SN, Marder G 1990. Tasas de infección toxoplasmica en el hombre y su relación con los animales domesticos en la ciudad de Corrientes. Vet Arg 68: 518-522.

White C 1973. Statistical Methods in Serum Surveys, p. 19-32. In JR Paul, C White (eds), Serological Epidemiology, Academic Press, New York.

Wilson MB, Nakane PK 1978. Antibody conjugated to horse-radish peroxidase, p. 215-224. In W Kanpp, K Holubar, G Wick (eds), Immunofluorescence and related staining technique, . Elsevier North Holland Biomedical Press, Amsterdam.

Yamamoto YI, Hoshino-Shimizu S, Camargo ME 1991. A novel IgM indirect hemagglutination test for the serodiagnosis of acute toxoplasmosis. J Clin Lab Anal 5: 127-132. 